Table 1. Adjusted Versus Unadjusted Effect Estimates, Highlighting the "Table 2 Fallacy"

\begin{tabular}{lcc}
\hline Covariate & $\begin{array}{c}\text { Adjusted Odds Ratio } \\
(95 \% \mathrm{Cl})\end{array}$ & $\begin{array}{c}\text { Crude Unadjusted Odds } \\
\text { Ratio }(95 \% \mathrm{Cl})^{\mathrm{a}}\end{array}$ \\
\hline $\begin{array}{l}\text { Antibiotics in the } \\
\text { previous 7 days }\end{array}$ & $7.65(3.13-19.42)$ & $3.567(1.79-6.97)$ \\
\hline Immunocompromised & $3.51(1.44-8.81)$ & $1.716(0.910-3.16)$ \\
\hline Abnormal chest x-ray & $2.54(1.07-5.98)$ & $5.174(2.99-9.42)$ \\
\hline $\begin{array}{l}\text { Discharge diagnosis of } \\
\text { pneumonia }\end{array}$ & $63.3(15.1-445)$ & $40.55(13.20-167.85)$ \\
\hline
\end{tabular}

Note. $\mathrm{Cl}$, confidence interval.

${ }^{a}$ Crude unadjusted odds ratios were calculated in R studio software with the EpiTools package (R Foundation for Statistical Computing, Vienna, Austria).

reduce bias, and a statistically significant one with a small effect size may have trivial impact on bias.

Acknowledgments. None.

Financial support. No financial support was provided relevant to this article.
Conflicts of interest. All authors report no conflicts of interest relevant to this article.

\section{References}

1. Li J, Kang-Birken SL, Matthews SK, Kenner CE, Fitzgibbons LN. Role of rapid diagnostics for viral respiratory infections in antibiotic prescribing decision in the emergency department. Infect Control Hosp Epidemiol 2019;40:974-978.

2. Fleming-Dutra KE, Hersh AL, Shapiro DJ, et al. Prevalence of inappropriate antibiotic prescriptions among US ambulatory care visits, 2010-2011. JAMA 2016;315:1864-1873.

3. Antibiotic/antimicrobial resistance biggest threats and data. Centers for Disease Control and Prevention website. https://www.cdc.gov/drugresistance/ biggest-threats.html. Published 2019. Accessed December 1, 2019.

4. Westreich D, Greenland S. The table 2 fallacy: presenting and interpreting confounder and modifier coefficients. Am J Epidemiol 2013;177: 292-298.

5. Bandoli G, Palmsten K, Chambers CD, Jelliffe-Pawlowski L, Baer RJ, Thomspon CA. Revisiting the table 2 fallacy: a motivating example examining preeclampsia and preterm birth. Paediatr Perinat Epidemiol 2018; 32:390-397.

\title{
Role of telehealth in outbreaks-Where the classical healthcare systems fail
}

\author{
Ata Mahmoodpoor MD, FCCM ${ }^{1}$ (1), Mohammad Amin Akbarzadeh ${ }^{2}$ (ㄷ), Sarvin Sanaie MD, $\mathrm{PhD}^{3}$ (1) and \\ Mohammad-Salar Hosseini ${ }^{4}$ \\ ${ }^{1}$ Department of Anesthesiology and Critical Care Medicine, Faculty of Medicine, Tabriz University of Medical Sciences, Tabriz, Iran, ${ }^{2}$ Student Research \\ Committee, Tabriz University of Medical Sciences, Tabriz, Iran, ${ }^{3}$ Aging Research Institute, Tabriz University of Medical Sciences, Tabriz, Iran and ${ }^{4}$ Research \\ Center for Evidence-Based Medicine, Tabriz University of Medical Sciences, Tabriz, Iran
}

To the Editor-Outbreaks impose massive burdens on healthcare systems. For example, $>510$ deaths were reported among the healthcare workers (HCWs) during the last Ebola outbreak, and by February 24, 2020, >3,300 HCWs had been infected with SARS-Cov-2 in China alone. ${ }^{1,2}$ This issue represents the essence of outbreaks - a mass of patients who require prompt medical care. Under these conditions, the classical healthcare system cannot manage the large number of COVID-19 patients immediately and effectively. ${ }^{3}$

Telehealth provides vital services through the application of information and communication technologies for each stage of managing diseases, research, and continuing education. ${ }^{4}$ With telecommunication, visits are more economical and preserve government, community, and family resources, which are already limited during global outbreaks. It can be used to provide rapid diagnosis, and it enables caregivers to act quickly. ${ }^{5}$ With the appropriate utilization of telecommunication, it is possible bring access to medicine and concurrent therapy in hard-to-reach regions and

Author for correspondence: Mohammad-Salar Hosseini, E-mail: hosseini.msalar@ gmail.com

Cite this article: Mahmoodpoor A, et al. (2020). Role of telehealth in outbreaksWhere the classical healthcare systems fail. Infection Control \& Hospital Epidemiology, 41: 992-994, https://doi.org/10.1017/ice.2020.120 communities, facilitating direct-to-patient or specialty consultation services from a distance, which is cost-effective and improves the efficacy of follow-up. ${ }^{6}$

The benefits of implementing telehealth in outbreaks comprise 8 main areas of focus:

1. The available admission capacity and the number of HCWs are limited, and the hospital infrastructure may not be adequate to serve all patients. Figure 1 presents an optimum model of telemedicine that could aid medical and social management during an outbreak.

2. The more patients referred to healthcare facilities, the more HCWs are at risk of being infected. Also, most referrals are unnecessary and merely increase the load on the healthcare system. Furthermore, people referred to hospitals are at an incredibly higher risk of infection. More than $40 \%$ of the infections are assumed to be hospital related, though they should be quite simple to prevent (Fig. 1). ${ }^{7}$

3. In affected regions, most healthcare facilities are dedicated to the management of COVID-19 patients. Therefore, noncritical patients (like patients with chronic and metabolic disorders such as hypertension, diabetes, and hyperlipidemia) have a lower priority. As a result of self-isolating, these people, mostly the older adults, cannot attend the healthcare units. A 


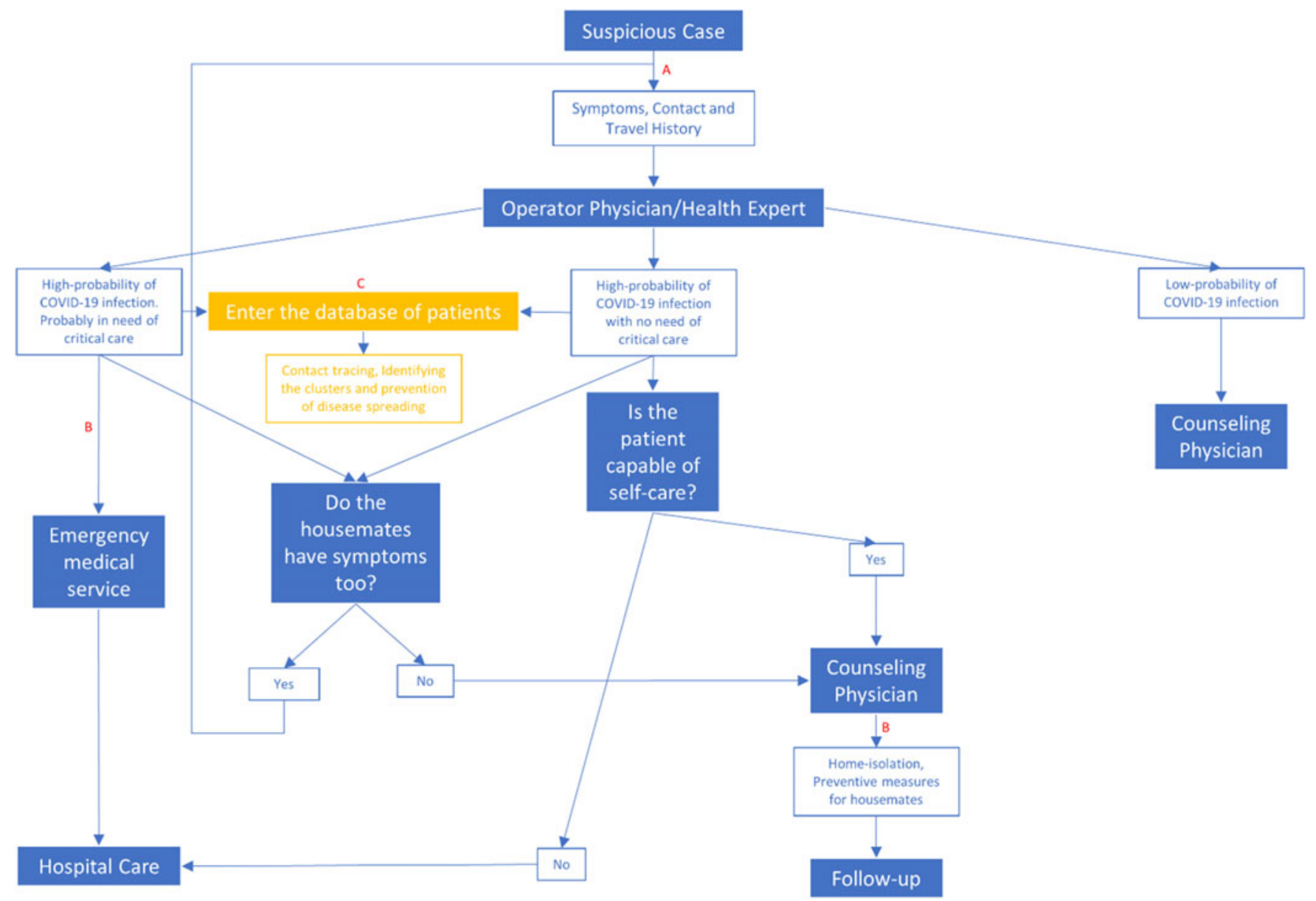

Fig. 1. Algorithm of telemedicine application during the COVID-19 outbreak. This model optimizes the use of telehealth, telemedicine, and emergency medical services. This model obviates unnecessary referrals, reduces the load of the healthcare system, and systematizes hospital care. Axis 'A' displays the first application of telemedicine, which omits unnecessary referrals by implementing a diagnostic role. Axis 'B' provides the required care under the supervision of a physician without the need for an in-person visit. Axis ' $C$ ' contributes to limiting the outbreak and social management in the outbreak setting.

remote follow up and visit of these patients could prevent the consequences of poorly controlling the diseases.

4. During a worldwide health emergency, some people with less mobility (ie, disabled patients) may not even be able to access the nearest local care center. Telehealth can facilitate assistance in their location while simultaneously providing clinicians instant access to their medical records.

5. In many countries, medical schools and universities are among the closed establishments. The duration of this shutdown is unknown, and medical education should be continued through the online and virtual classes. Medical conferences and gatherings could be scheduled likewise.

6. Proper use of popular social networks could significantly aid in prevention by effectively educating the populace.

7. Using a referral system based on telehealth can provide immediate access to medical records of infected patients. These records could be analyzed to identify the cluster "hot spots."

8. Developing a network could help clinicians discuss special cases and share the latest findings and their evidence-based experiences regarding the daily advances and findings related to the disease. The network provides an advantage during health crises by enabling rapid and efficient responses.
However, adopting telemedicine or telehealth may be difficult because of costs, the challenges of high technology, lack of awareness, limited access to high-speed internet, reimbursement problems, and availability of technical support are the issues that may slow down the application of telehealth. ${ }^{9}$ These barriers are present in both developing and developed countries. In developing countries, the primary obstacle is most likely the implementation of new technology (eg, high-speed internet), and in developed countries, the most concerning matter is the need for new behavioral patterns and how to develop them. The essentials are easy to implement, and with advantages such as improved investigations, disease control, clinical case management, and information enhancement, societies can benefit from the telehealth method. In outbreaks, it is important to rapidly increase treatment units, to expand their reach through telehealth, and to develop and disseminate appropriate treatment methods for communities, and a great demand for support and assistance is expected. Such interventions using telehealth could eventually connect all healthcare personnel across the world, which could be very beneficial during a pandemic. At this point, telehealth and telemedicine comprise one of the few reliable strategies that could intervene when the classical system of healthcare becomes ineffective. 


\section{Acknowledgments.}

Financial support. No financial support was provided relevant to this article.

Conflicts of interest. All authors report no conflicts of interest relevant to this article.

\section{References}

1. Elston J, Cartwright C, Ndumbi P, Wright J. The health impact of the 201415 Ebola outbreak. Public Health 2017;143:60-70.

2. Wang J, Zhou M, Liu F. Exploring the reasons for healthcare workers infected with novel coronavirus disease 2019 (COVID-19) in China. J Hosp Infect 2020; pii: S0195-6701(20)30101-8. doi: 10.1016/j.jhin.2020.03.002.

3. Ji Y, Ma Z, Peppelenbosch MP, Pan Q. Potential association between COVID-19 mortality and health-care resource availability. Lancet Global Health 2020;8(4):e480. doi: 10.1016/S2214-109X(20)30068-1.
4. Weinstein RS, Lopez AM, Joseph BA, et al. Telemedicine, telehealth, and mobile health applications that work: opportunities and barriers. Am J Med 2014;127:183-187.

5. Tuckson RV, Edmunds M, Hodgkins ML. Telehealth. N Engl J Med 2017;377:1585-1592.

6. Dorsey ER, Topol EJ. State of telehealth. N Engl J Med 2016;375:154-161.

7. Wang D, Hu B, Hu C, et al. Clinical characteristics of 138 hospitalized patients with 2019 novel coronavirus-infected pneumonia in Wuhan, China. JAMA 2020;323:1061-1069.

8. Wood CS, Thomas MR, Budd J, et al. Taking connected mobile-health diagnostics of infectious diseases to the field. Nature 2019;566:467-474.

9. Scott Kruse C, Karem P, Shifflett K, Vegi L, Ravi K, Brooks M. Evaluating barriers to adopting telemedicine worldwide: a systematic review. J Telemed Telecare 2018;24:4-12.

\title{
Antibiotic price rise and antibiotic stewardship programs-Stimulus or discouragement?
}

\author{
Felipe Francisco Tuon $\mathrm{PhD}^{1}$ (1), Joao Paulo Telles $\mathrm{MD}^{2}$, Juliano Gasparetto $\mathrm{PhD}^{3}$ and Tiago Zequinão Pharm ${ }^{1}$ \\ ${ }^{1}$ Laboratory of Emerging Infectious Diseases, Pontifícia Universidade Católica do Paraná, Curitiba PR, Brazil, ${ }^{2}$ Infectious Diseases Department, AC Camargo \\ Cancer Center, São Paulo, SP, Brazil and ${ }^{3}$ School of Medicine, Pontifícia Universidade Católica do Paraná, Curitiba PR, Brazil
}

To the Editor-An antimicrobial stewardship program (ASP) can be defined as the set of actions performed in hospitals for the rational use of antibiotics; reduction of adverse events, dosage errors, and appearance of multidrug-resistant bacteria; and shortening of length of hospital stay. The performance of the professional team implementing these programs, including clinical pharmacists, physicians and nurses, is associated with reduced in-hospital mortality rates. ${ }^{1}$ Managed antimicrobial administration programs shorten the length of hospital stay and reduce costs associated with the use of these medications.

Antibiotics account for a considerable cost in hospital bills, accounting for almost $20 \%$ of drug costs in Brazilian intensive care units. ${ }^{2}$ The usage policy is a controversial subject because it varies among hospitals. De-escalation of therapy and a switch from an intravenous to oral regimen, if it does not cause harm and demonstrates a safe strategy, can have an important outcome. ${ }^{3}$

ASPs are safe and cost-effective, an approach important particularly in developing countries. ${ }^{4}$ We demonstrated an estimated savings of US\$514,831 as a result of the implementation of the stewardship program in 2 institutions. These numbers encourage hospital administrators because there is always pressure to reduce costs. However, since the implementation of the ASPs in our hospitals, the price of medications has increased disproportionately. Figure 1 clearly shows the disproportionate increasing in the price of antimicrobials purchased by our group of hospitals in relation to various value indices.

The cost of some antibiotics increased $>500 \%$ over a period of 1 year (Fig. 1). This situation has led to a series of measures within

Author for correspondence: Felipe F. Tuon, E-mail: flptuon@gmail.com

Cite this article: Tuon FF, et al. (2020). Antibiotic price rise and antibiotic stewardship programs-Stimulus or discouragement?. Infection Control \& Hospital Epidemiology, 41: 994-995, https://doi.org/10.1017/ice.2020.125

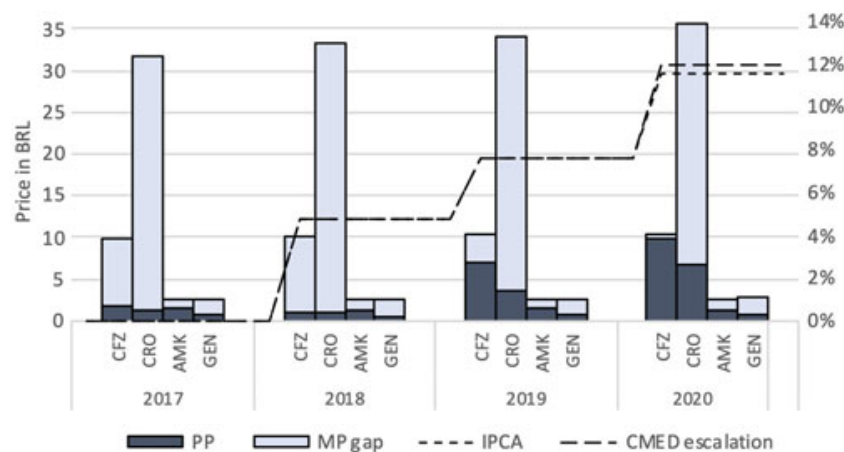

Fig. 1. Prices of 2 essential antibiotics, cefazolin (CFZ) and ceftriaxone (CRO), in the last 4 years, showing increasing costs, instead of aminoglycosides, amikacin (AMK), and gentamicin (GEN). The dark-gray columns indicate the price of each antibiotic and the light-gray columns indicate the maximum price allowed in Brazil. The dashed lines are the market index, IPCA for consumer prices, and CMED for drugs. Note. PP, purchase price; MP, maximum price; IPCA, Brazilian Broad Consumer Price Index; CMED, Brazilian Drugs Market Regulation Chamber.

our hospitals to contain costs to maintain the viability of our public hospital. We changed the surgical prophylaxis protocol from cefazolin to clindamycin. We implemented an active ASP for switching from an intravenous to an oral regimen as well as a de-hospitalization program (ie, outpatient antimicrobial therapy with oral and intravenous options). This cost crisis could be a stimulus to improve the ASP, but it will be discouraging if a cost reduction is not achieved.

In Brazil, the pricing of medicine is regulated by an agency called CMED (cf, Drug Market Regulation Chamber), which defines the maximum drugs prices that can be sold and the readjustment rates (Law no. 10.742, October 6, 2003). Annual escalation is calculated using an equation which IPCA (Broad 